\title{
Agent-based modelling of genetic deafness propagation under various sociodemographic conditions
}

\author{
S.A. Lashin ${ }^{1,2 *}$, Yu.G. Matushkin ${ }^{1,2}$, A.A. Smirnova ${ }^{1}$, G.P. Romanov ${ }^{3,4}$, O.L. Posukh ${ }^{1,2}$ \\ ${ }^{1}$ Institute of Cytology and Genetics SB RAS, Novosibirsk, Russia \\ ${ }^{2}$ Novosibirsk State University, Novosibirsk, Russia \\ ${ }^{3}$ MK Ammosov North-Eastern Federal University, Yakutsk, Russia \\ ${ }^{4}$ Yakut Science Centre of Complex Medical Problems, Yakutsk, Russia \\ *e-mail:lashin@bionet.nsc.ru
}

Key words: deafness, agent based simulations, population genetics, social demography

Motivation and Aim: Genetic deafness is a heterogeneous disability with different inheritance patterns, the most common form of which is a recessive deafness caused by mutations in connexin-26 gene (Cx26, GJB2). High frequency of Cx26-associated deafness in European and American populations was previously assumed to be caused by combined effects of assortative mating tradition among deaf people based on linguistic homogamy (sign language) and relaxed selection against deafness [1, 2]. Although there were some attempts to study interrelations between social and genetic factors in spreading of genetic deafness using mathemtical modelling and computer simulations [2], there is still lack of both methodological basis and software tools for this task. In this study, we present an agend-based model "Population Genetics of Deafness" (PGD) along with its software implementation and biologically relevant simulation results.

Methods and Algorithms: Agent-based model "Population Genetics of Deafness" (PGD) describes propagation of genetic deafness in human populations through generations. The model takes into account social factors of mating, such as: choice-by-phenotype and sign language. Model allows simulating either simple mendelian (one-, two-, or multi-locus, bi- or multi-allelic) or multifactorial genotype-phenotype interactions. The software implementation of PGD is based on Diploid Evolutionary Constructor framework [3].

Results: We have considered series of simulations (20-fold for each scenario) of deafness propagation varying the following parameters: initial population size (including frequencies of each cohorts), sign language assortativity and phenotypic assortativity. Simulation results are counter-intuitive versus [1, 2]: increase of assortative mating in deaf subpopulation led to decrease of the frequency of "deaf" alleles after 25 generations. It is especially true for the cases when increased assortativity is associated with relatively high significance of sign language proficiency and low number of its carriers in initial population.

Conclusion: We have presented an agent-based model "Population Genetics of Deafness" (PGD) and its software implementation. Simulations performed with PGD have shown counter-intuitive behavior of genetic deafness propagation associated with assortative mating and other social factors like sign language expansion in populations. Acknowledgements: The study was supported by the Budget Project 0324-2018-0017.

References

1. Nance W.E. et al. (2000) Lancet. 356(9228):500-501.

2. Nance W. E., Kearsey M. J. (2004) American Journal Human Genetics. 74(6):1081-1087.

3. Lashin S.A., Matushkin Yu.G. (2013) Multi-layer computer models of gene network evolution in diploid populations. FEBS Journal. 280:563-563. 